\title{
Modelling the effect of lockdown
}

\author{
Hideto Kamei*, Akihiro Sato ${ }^{\dagger}$
}

2020-5-6

\section{Abstract}

This note models the effect of the lockdown during the first wave of COVID19. We use SEIR type of model with a certain time lag between infection and becoming infectious. Firstly we compare the timing of the change of the coefficient of infection, growth rate of confirmed cases corresponds to the change of mobility index, and secondly we assume the change of the coefficient of infection, activity index $\beta$ (analogous to $R_{0}$ ) and fit the parameter to reproduce the actual number of confirmed cases. Finally, we assume that the activity index $\beta$ is proportional to the square of the mobility and fit the parameters. The curves in various cuontries fits reasonably well in any cases, but estimating $\beta$ from various parameters (including temperature) remains as an important task.

\section{Introduction}

Due to the worldwide pandemic of COVID-19, many countries are trying to slow down the spreading of the virus through lockdown of the cities. At the time of writing this $(2020 / 4 / 29)$ some has already achieved dramatic decrease in the number of patients, while most of them are still struggling, therefore it is crucial to evaluate the effect of the lockdown, equivalently, to estimate the time the lockdown is needed.

Therefore, we will try to model the dynamics for the number of patients, considering the effect of lockdown. We have roughly two different type of models which could explain the dynamics, one is ordinary SEIR type of model as in [1], in which transfer from one state to another is proportional to the number of original state, and another is SEIR type of model which includes the effect of time delay[2]. In this model, once someone gets infected, they will become contagious and later quarantined after fixed period of time ${ }^{1}$. Using actual num-

\footnotetext{
${ }^{*}$ hidetokamei@gmail.com

†Yokohama City University Department of Data Science, Graduate Graduate School of Data Science, ahsato@yokohama-cu.ac.jp

${ }^{1}$ In reality, we know that the time infected person becomes contagious is rather fixed[3], and also it will take a while since patients go to a doctor till they receive the result of PCR testing. Therefore we expect the model with time delay will describe the dynamics better.
} 
ber of patients[5] and the activity data from mobile phone[6], we wish to know which type of model better describes the reality.

In the second section, we briefly describe the structure of two types of models, and in the third section we will see the overview of the model and fitting of the models with the real data. In the final section we will examine the result and possible future developments.

\section{Model}

Let me describe two types of models of contagion. The ordinary SEIR type of model we consider here consists of 5 (or 4 for simplicity) states. $S$ : susceptible(not infected), $E$ : Exposed(infected but not yet contagious) $I$ :Infected(infected and contagious) $Q$ :Quarantined(infected but separated from others) $R$ :Recovered(infected but recovered and no more contagious). Naturally the population of those 5 states adds up to the total number of population $N$. We assume that the transfer from state $S$ to state $E$ is proportional to $S$ and $I$, because the infection happens via the contact of people in these two states. We further assume the transfer from $E$ to $I$ is proportional to the number of $E$ (therefore it is somewhat similar to the decay of a particle to another state), and transfer from $I$ to $Q$ and $I$ to $R$ are also proportional to the number of people in state $I$.

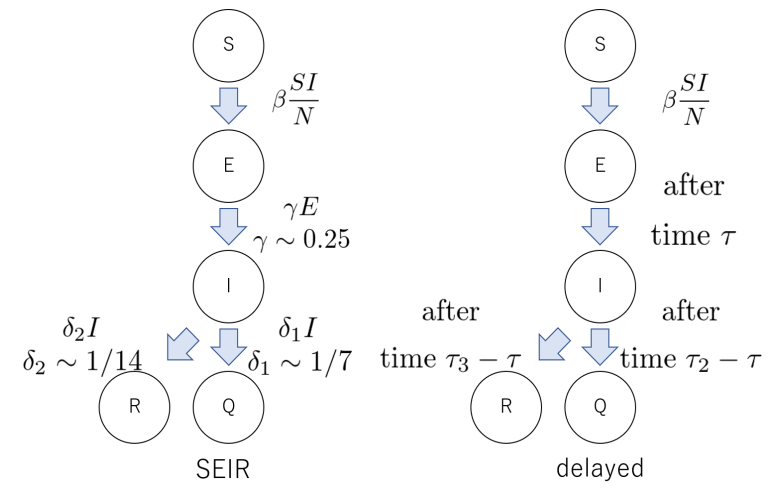

Figure 1: Transfer between states in SEIR model and delayed model.

Therefore, denoting the number of people in that particular state as the same letter, we would have a combination of equations 


$$
\begin{aligned}
\dot{S} & =-\beta(t) \frac{S(t) I(t)}{N} \\
\dot{E} & =\beta(t) \frac{S(t) I(t)}{N}-\gamma E(t) \\
\dot{I} & =\gamma E(t)-\left(\delta_{1}+\delta_{2}\right) I(t) \\
\dot{Q} & =\delta_{1} I(t) \\
\dot{R} & =\delta_{2} I(t) .
\end{aligned}
$$

The parameter in the model can be estimated by following clinical consideration; Since it is known one will be infectious after 3 to 5 days after the infection, the time scale for transferring from $\mathrm{E}$ to I can be estimated to be around $4^{2}$. Time scale for transfer from $I$ to $Q$ can be estimated to be around 1 week considering the situation in Japan[4], but expected to vary from country to country. The transfer parameter $\delta_{2}$ from state $I$ to state $R$ is expected to be around $1 / 14$, because it is considered it takes about two weeks for COVID-19 to be cured(to become not infectious). Another type of model is SEIR type of model with delay in time and the equations are given as below.

$$
\begin{aligned}
& \dot{S}=-\beta(t) \frac{S(t)}{N} I(t) \\
& \dot{E}=\beta(t) \frac{S(t)}{N} I(t)-\beta(t-\tau) \frac{S(t-\tau)}{N} I(t-\tau) \\
& \dot{I}=\beta(t-\tau) \frac{S(t-\tau)}{N} I(t-\tau)-a \beta\left(t-\tau_{2}\right) \frac{S\left(t-\tau_{2}\right)}{N} I\left(t-\tau_{2}\right)-(1-a) \beta\left(t-\tau_{3}\right) \frac{S\left(t-\tau_{3}\right)}{N} I\left(t-\tau_{3}\right) \\
& \dot{Q}=a \beta\left(t-\tau_{2}\right) \frac{S\left(t-\tau_{2}\right)}{N} I\left(t-\tau_{2}\right) \\
& \dot{R}=(1-a) \beta\left(t-\tau_{3}\right) \frac{S\left(t-\tau_{3}\right)}{N} I\left(t-\tau_{3}\right) .
\end{aligned}
$$

In reality, once you get infected with coronavirus, becoming symptomatic and being tested positive happens after certain amount of time rather than happening at certain probability. Thus, we have a heuristic reason to believe the latter model better describes the reality. In the later discussion in this note, we will focus on the delayed SEIR model and leave the comparison between the two models for appendix. If transfer from $S$ to $E$ happens at time t, transfer from state $E$ to state $I$ happens at time $t+\tau$, where $\tau \sim 4$, and assuming that state $I$ will proceed to state $Q$ with probability $a$ and to state $\mathrm{R}$ with probability $(1-a)$, we further assume that the transfer from state $I$ to state $Q$ will happen at time $t-\tau_{2}$ and $I$ to $R$ at time $t-\tau_{3}$.

\footnotetext{
${ }^{2}$ The time from infection till one becomes mptomatic is around 5 days as in the study in Diamond Princess[3] and it is also known that one can be infectious $1 \sim 2$ days before one becomes symptomatic[8]. Therefore time scale for this process is around 4 and $\gamma$ should be around 0.25 .
} 


\section{Data and anaysis}

After brief description on the datasets, we will perform three different data analysis to know which of the two models above describe the dynamics of confirmed cases better including the effect of lockdown.

\subsection{Data}

We use the data for the confirmed cases from ourworlddata.org[5] and mobility data from apple mobility report[6]. The apple mobility report records how many times people searched for certain path using apple map, and the number of search is counted for car, walk and transit respectively.

\subsection{First Analysis}

In the first analysis, we wish to know the time lag from infection to quarantine $\left(\tau_{2}\right)$ heuristically. If the value of $\beta$ and $S$ is constant, the growth rate $^{3}$ of $I$ should be proportional to $\beta$. Therefore, naively, we could expect that the growth rate of $Q$ should be the same, so we might expect that the value of $\beta$ and growth rate of $\dot{Q}(=$ daily confirmed cases $)$ is proportional. We have changed the value of $\tau_{2}$ and performed the linear regression of growth rate of $\dot{Q}(t)$ with respect to $\beta\left(t-\tau_{2}\right)$, and computed $\mathrm{p}$-value for each regression.

There are roughly 5 countries which has already achieved the drastic decrease in the number of confirmed cases and data is available, Australia, Austria, Czech, Norway and Switzerland. We would focus on those 5 countries. Among those, please take a look at figures for Australia and Austria below. First figure shows the number of confirmed cases, and the second shows the growth rate of the number of confirmed cases and activity index taken by $[6]^{4}$. In the third figure, growth rate in confirmed cases and activity 12 days before was plotted.

You can see that the growth rate changes in response to the change of activity index with around 10 days of delay. We have also performed linear regression on growth rate of confirmed cases and activity index, changing the time lag $\tau$. As a result, we will have p-value for the regression and we have plotted the $\log (\mathrm{p}$-value) in the Figure 8. If we interpret the location of minimum p-value as the most probable model ${ }^{5}$, we can conclude $\tau \sim 12$.

\subsection{Second Analysis}

In the next step, let's perform two types of simulation I have described, SEIR type model and SEIR type model with delay. In this part, we simplify the analysis for ease of analysis. Here we ignore the state $R$ because it is a bit hard

\footnotetext{
${ }^{3}$ We computed the slope of confirmed cases with respect to time as the growth rate.

${ }^{4}$ In the original database, the amount of search people performed through apple map for walk, drive, transit are recorded and we just took the average of these indices as activity index.

${ }^{5}$ If possible we should compare the model of different $\tau$ by free energy or BIC, but we don't have time to do that.
} 


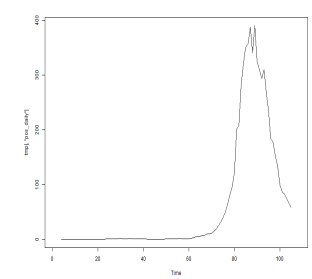

Figure 2: daily confirmed cases in Australia

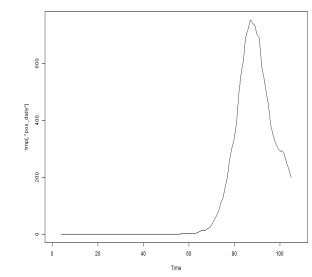

Figure 5: daily confirmed cases in Austria
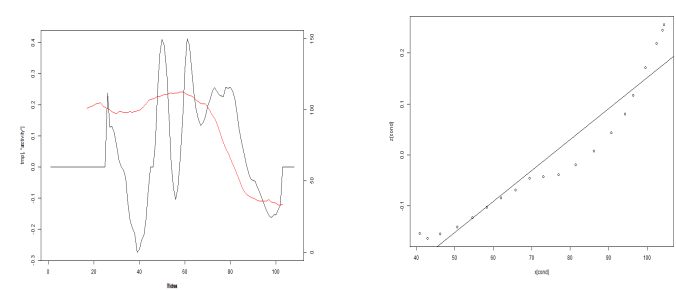

Figure 3: activity index and growth rate of confirmed cases in Australia

Figure 4: activity index $(12$ days before) and growth rate of confirmed cases in Australia
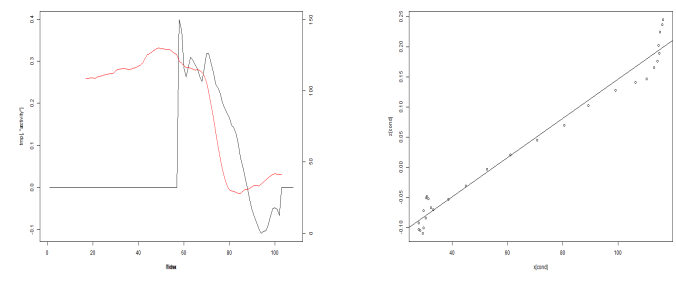

to know the ratio of patients who will not quarantined but be cured without any treatment.

For the former model, we have variables the number of people for $S, E, I, Q$, and total number of people $N$, and transfer coefficients $\gamma, \delta$ are given by medical consideration. We will fit the model to the data by tuning $I_{0}$ ( $I$ at certain time), and the value of $\beta$. We naively expect the value of $\beta$ to be proportional to activity index, but then it will not match the observation. We then assume that the time dependence of $\beta$ is similar to that of activity index. That is, as in Figure 9, we first approximate the dynamics of activity index as constant for $t<t_{1}$ and $t>t_{2}$, and linearly decrease for $t_{1}<t \leqq t_{2}$. We then assume that $\beta$ is constant for $t<t_{1}$ and $t>t_{2}$, and linearly decrease for $t_{1}<t \leqq t_{2}$.

In the delayed SEIR model, we instead have $N$ and $\tau$ from clinical consideration, because it is known that the time period from infection till one gets infectious is considered to be around 4 days. We then fit the model to daily confirmed cases and obtain $\tau_{2}, \beta_{0}, \beta_{1}, I_{0}$.

If we wish to see $\log (\dot{Q}), I_{0}$ corresponds to the intercept of the line, $\beta_{0}$ corresponds to the slope of the line, $\tau_{2}$ corresponds to the time of lockdown, and $\beta_{1}$ corresponds to the slope of the line after lockdown, so it is not so hard to estimate the value of the parameters. Here we adjust those parameters by hand and saw the value of $\dot{Q}$ for actual and model. Here we show plot of those together with the plot of $\beta$.

For Australia, the parameter for the delayed model is, $\beta_{0}=1.0, \beta_{1}=0.04 \beta_{0}, \tau=$ 
Figure 8: $\log (\mathrm{p}$-value) of linear regression of growth rate of confirmed cases with respect to activity index, changing time lag $\tau$
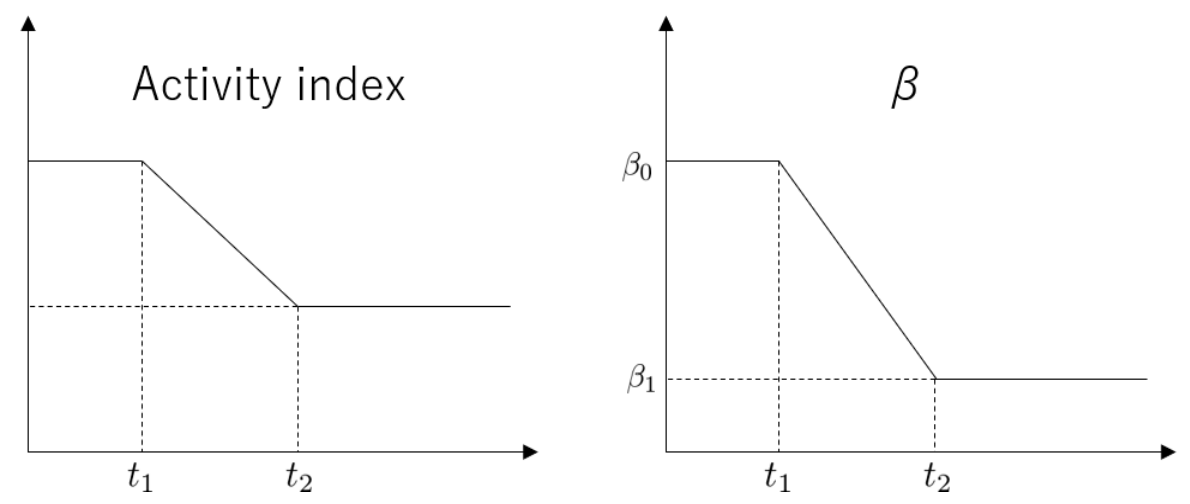

Figure 9: Time dependence of activity and the value of $\beta$.

$4, \tau_{2}=12, I_{0}=0.01, t_{0}=2 / 2, t_{1}=3 / 12, t_{2}=3 / 23$. For Austria, the parameter for the delayed model is $\beta_{0}=0.7, \beta_{1}=0.07 \beta_{0}, \tau=4, \tau_{2}=14, I_{0}=6, t_{0}=$ $3 / 1, t_{1}=3 / 7, t_{2}=3 / 15$. Here $t_{0}$ denotes the time when the number of confirmed cases became more than or equal to 10 for the first time (and we set the day to $t=0$ when we draw the graph for the number of patients). Considering the fact that the value of the activity became $20 \%$ of that due to lockdown in Australia as in Figure 10, and $30 \%$ in Austria as in Figure 13, the decline in $\beta$ in Figure 11, Figure 14 were much more drastic compared to the decline in the value of the activity. From the fact that the ratio of $\beta_{1}$ to $\beta_{0}$ is close to the square of ratio of activity index, we can guess that the value of $\beta$ might just be proportional to square of activity index ${ }^{6}$. SEIR model with time delay managed

\footnotetext{
${ }^{6}$ That actually makes sense, because if we assume for example, only $20 \%$ of people go out and the rest stay home, both $S$ and $I$ will be multiplied by $20 \%$ and effectively $\beta$ becomes square of $20 \%$.
} 

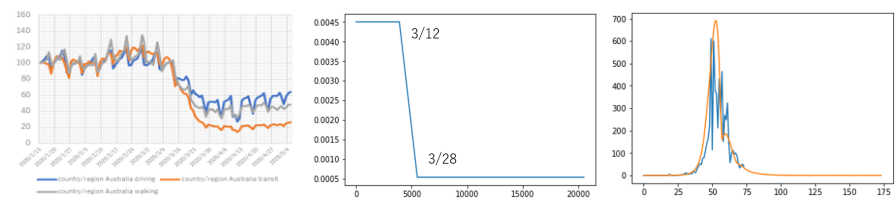

Figure 10: value of mo- Figure 11: value of $\beta$ in Figure 12: $\dot{Q}$ for acbility in Australia Australia tual and delayed model
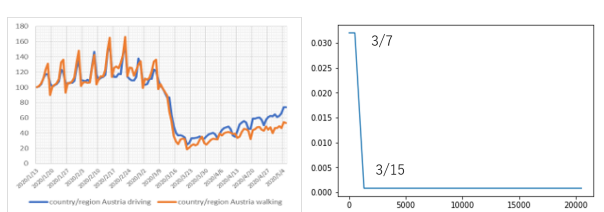
in Australia

Figure 13: value of mo- Figure 14: value of $\beta$ in Figure 15: $\quad \dot{Q}$ for acbility in Austria Austria tual and delayed model in Austria

to reproduce the real data as in Figure 12, and Figure 15. Please refer to the appendix for the data fitting for SEIR model without delay.

Let's try to fit the delayed model for the rest of the countries which experienced successful lockdown. With respect to each country, the estimated parameters would be as in table 1 . The error between real value and theoretical value from delayed SEIR model is measured by $\sum_{\text {days }}\left(\log \left(\dot{Q}_{\text {real }}\right)-\log \left(\dot{Q}_{\text {model }}\right)\right)^{2}$.

\begin{tabular}{|c||r|r|r|r|r|r|r|r|r|r|}
\hline country & $\beta_{0}$ & $\beta_{1} / \beta_{0}$ & $\tau$ & $\tau_{2}$ & $I_{0}$ & $t_{0}$ & $t_{1}$ & $t_{2}$ & $t_{2}-t_{1}$ & error \\
\hline \hline Japan & 0.3 & 0.3 & 4 & 11 & 0.8 & $1 / 30$ & $3 / 24$ & $4 / 13$ & 20 & 66.9 \\
South Korea & 1.0 & 0.2 & 4 & 7.2 & 1.5 & $2 / 1$ & $2 / 19$ & $2 / 26$ & 7 & 35.3 \\
Czech Republic & 0.4 & 0.15 & 4 & 15 & 20 & $3 / 6$ & $3 / 8$ & $3 / 20$ & 12 & 4.6 \\
Norway & 0.2 & 0.17 & 4 & 20 & 100 & $3 / 1$ & $3 / 5$ & $3 / 12$ & 7 & 16.3 \\
Switzerland & 0.6 & 0.1 & 4 & 13 & 10 & $2 / 29$ & $3 / 8$ & $3 / 19$ & 11 & 4.1 \\
Singapore & 0.29 & 0.17 & 4 & 16 & 0.04 & $1 / 20$ & $3 / 19$ & $4 / 10$ & 22 & 649.4 \\
United States & 1 & 0.1 & 4 & 13.7 & 0.004 & $2 / 3$ & $3 / 8$ & $3 / 22$ & 14 & 334.0 \\
Luxembourg & 1.1 & 0.12 & 4 & 9 & 15 & $3 / 13$ & $3 / 5$ & $3 / 20$ & 15 & 3.0 \\
Vietnam & 1.2 & 0.15 & 4 & 5.5 & 0.08 & $2 / 5$ & $3 / 3$ & $4 / 1$ & 29 & 83.7 \\
\hline
\end{tabular}

Table 1: The parameters when we fit delayed SEIR model to the data.

The change of $\beta$ and the resulting plot for confirmed cases per day will be as in Figure $16 \sim$ Figure 51. The ratios of activity index before and after the lockdown are respectively roughly $30 \%$ for Czech, $40 \sim 50 \%$ for Norway, and $40 \%$ for Switzerland, and square of those numbers roughly correspond to the ratio of $\beta$ before and after lockdown, again for those countries. ${ }^{7}$

\footnotetext{
${ }^{7}$ It is also claimed from purely analysing the data in Japan in some twitter accounts[7].
} 

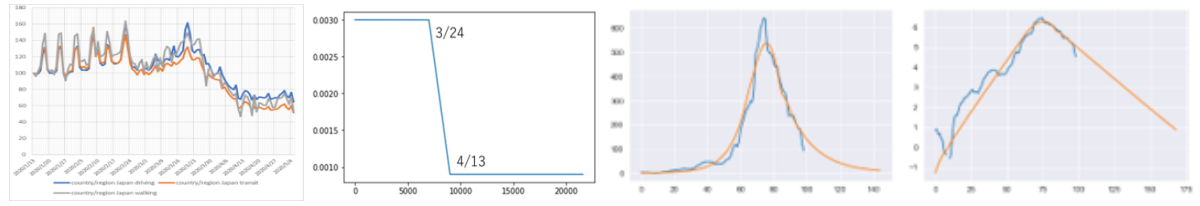

Figure 16: value of mo- Figure 17: value of $\beta$ in Figure 18: $\dot{Q}$ for ac- Figure 19: $\dot{Q}$ for acbility in Japan Japan tual and delayed model tual and delayed model in Japan in Japan (in log scale)
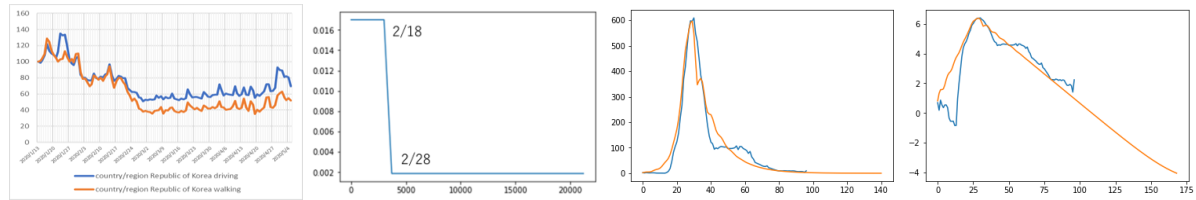

Figure 20: value of mo- Figure 21: value of $\beta$ in Fig bility in South Korea South Korea

Figure 22: $\dot{Q}$ for ac- Figure 23: $\dot{Q}$ for actual and delayed model tual and delayed model in South Korea in South Korea (in log scale)
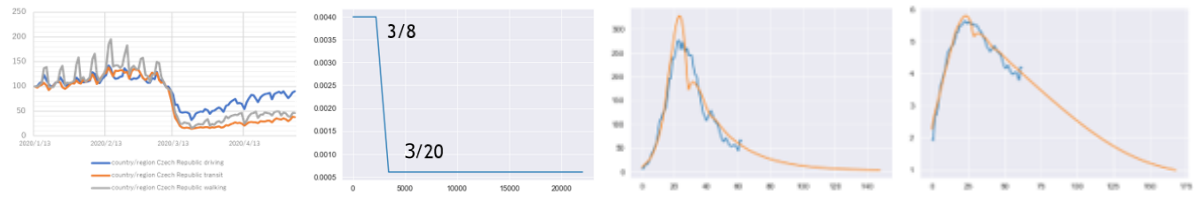

Figure 24: value of mo- Figure 25: value of $\beta$ in Figure 26: $\dot{Q}$ for ac- Figure 27: $\dot{Q}$ for acbility in Czech Republic Czech Republic tual and delayed model tual and delayed model
in Czech Republic in Czech Republic (in in Czech Republic $\quad$ in Czech
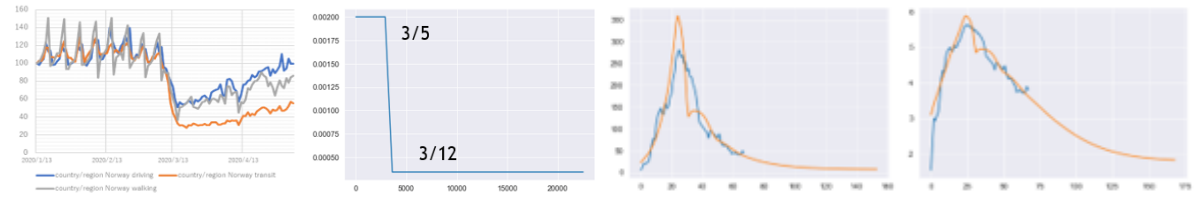

Figure 28: value of mo- Figure 29: value of $\beta$ in Figure 30: $\dot{Q}$ for ac- Figure $31: \dot{Q}$ for acbility in Norway Norway tual and delayed model tual and delayed model in Norway in Norway (in log scale) 

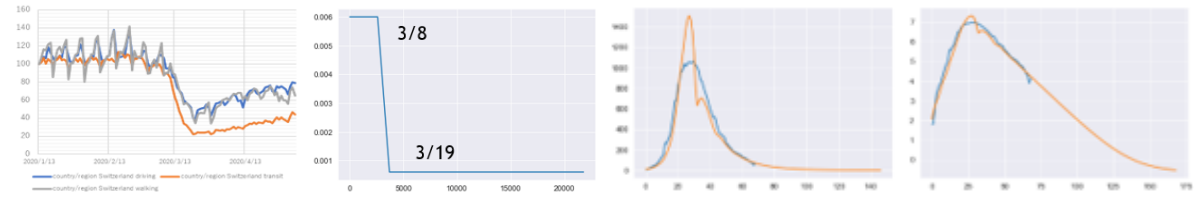

Figure 32: value of mo- Figure 33: value of $\beta$ in Figure 34: $\dot{Q}$ for ac- Figure 35: $\dot{Q}$ for acbility in Switzerland Switzerland $\quad$ tual and delayed model tual and delayed model in Switzerland in Switzerland (in $\log$ scale)
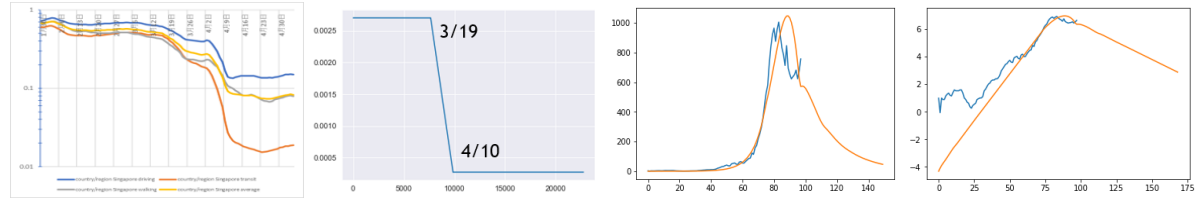

Figure 36: value of mo- Figure 37: value of $\beta$ in bility in Singapore Singapore

Figure 38: $\dot{Q}$ for ac- Figure 39: $\dot{Q}$ for actual tual and delayed model and delayed model in in Singapore Singapore (in log scale)
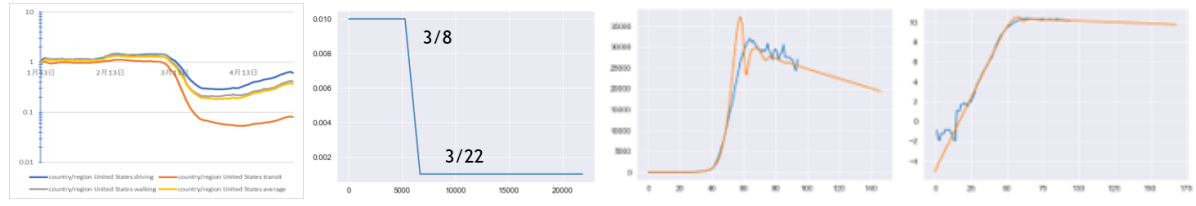

Figure 40: value of mo- Figure 41: value of $\beta$ in bility in United States United States

Figure 42: $\dot{Q}$ for ac- Figure $43: \quad \dot{Q}$ for actual and delayed model tual and delayed model in United States in United States (in log scale)
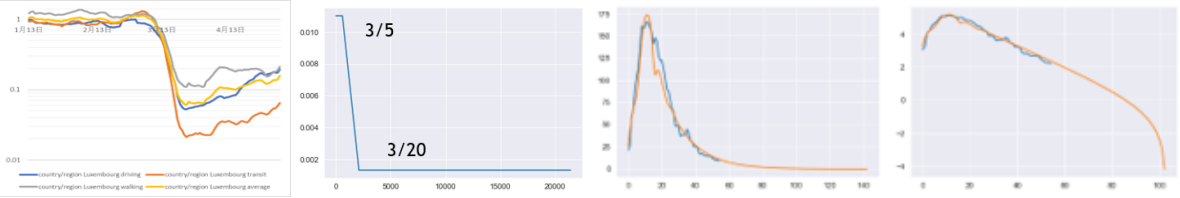

Figure 44: value of mo- Figure 45: value of $\beta$ in Figure $46: \quad \dot{Q}$ for ac- Figure $47: \quad \dot{Q}$ for acbility in Luxembourg Luxembourg tual and delayed model tual and delayed model in Luxembourg in Luxembourg (in log scale) 

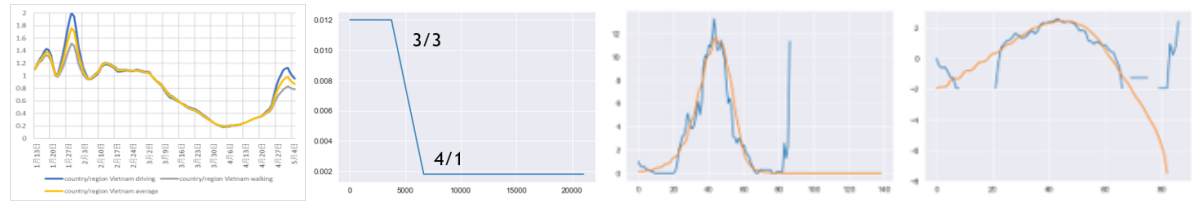

Figure 48: value of mo- Figure 49: value of $\beta$ in bility in Vietnam Vietnam

Figure 50: $\dot{Q}$ for ac- Figure 51: $\dot{Q}$ for actual
tual and delayed model and delayed model in in Vietnam

Vietnam (in log scale)

\subsection{Third Analysis}

We will improve the analysis in the previous subsection in two ways; Firstly, we have seen that the contact coefficient $\beta$ is roughly proportional to (mobility $)^{2}$, and then we reverse the logic and assume this proportionality relationship to derive the value of $\beta$. We will again use [6], and use two possible mobility indices, one is average of mobility indices of three transportation(driving, transit, walking $)^{8}$, and the other is mobility index for transit. Secondly, in the previous subsection, we have assumed fixed period of time one requires from the time of infection till the period one gets infectious, $\tau$, and from the time of infection till the period one gets tested and quarantined, $\tau_{2}$. Here instead we assume Weibull distribution for both time lag. From the analysis of [3], the distribution of the time period from the infection to starting to be symptomatic is, Weibull distribution with mean 6.4 and standard deviation 2.3. Considering the fact one becomes infectious $2 \sim 3$ days before one becomes symptomatic[8], we could pretend that the distribution of the time period from the infection to starting to be infectious is Weibull distribution with mean 4 and standard deviation 2.3. This amounts to Weibull distribution with parameter $k \sim 1.8$ and $\lambda \sim 4.5^{9}$. Furthermore, in case of Japan, the distribution from the time of becoming symptomatic and the time of getting tested is known [9], and adding typical time from infection till one gets tested, which is 6.4 days, to this distribution, the distribution of time from infection till getting tested can be approximated as another Weibull distribution, with $k=3.3$ and $\lambda=13.3$. We assume that for the corresponding distribution in other countries, the value of $k$ is 3 , and fit the $\lambda$ parameter for this distribution(denote as $\lambda_{2}$ ). As a result, fitting the curve by using $\beta=$ (mobility of public transportation) ${ }^{2}$ seems to be the best fit, so we will show the fit with that criterion and also show the curve from $\beta=$ (average of three types of mobility $)^{2}$. Moreover, we here assume $a=0$, considering generalization to $a=0$ will not change the dynamics qualitatively. The parameter for fitting is given as in table 2 .

\footnotetext{
${ }^{8}$ Note there is no theoretical background why we should take the average of the three values, since it is NOT weighted average of those

${ }^{9}$ Remember that Weibull distribution is $f(t)=(k / \eta)(t \eta)^{k-1} \exp \left\{-(t / \eta)^{k}\right\}$
} 

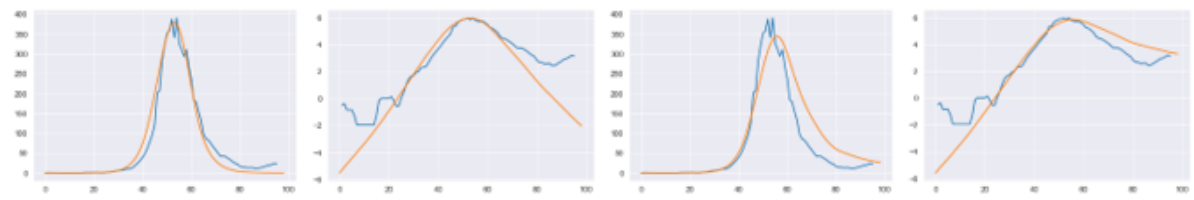

Figure 52: Fitting Figure 53: Fitting Figure 54: Fitting Figure 55: Fitting of $\dot{Q}$ for Australia of $\dot{Q}$ for Australia of $\dot{Q}$ for Australia of $\dot{Q}$ for Australia

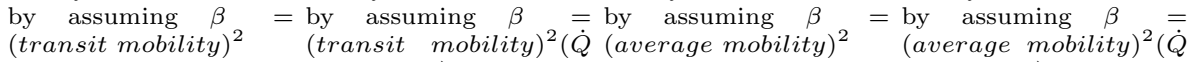

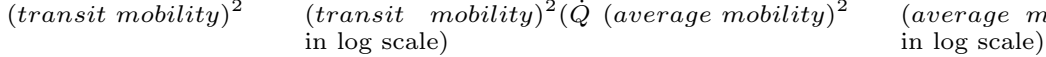
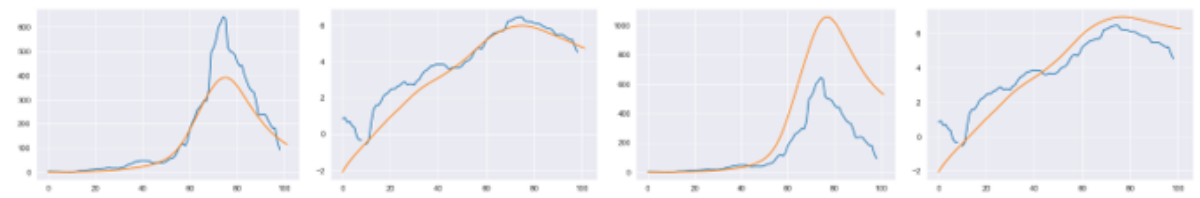

Figure 56: Fitting Figure, 57: Fitting Figure, 58: Fitting Figure 59: $\quad$ Fitting of $\dot{Q}$ for Japan of $\dot{Q}$ for Japan of $\dot{Q}$ for Japan of $\dot{Q}$ for Japan $\begin{array}{l}\text { by assuming } \beta \\ (\text { transit mobility })^{2}\end{array}=$ by assuming $\beta=$ (transit mobility $)^{2}\left(\begin{array}{l}\dot{Q} \\ \text { (average mobility })^{2}\end{array}=\underset{\text { (average mobility })^{2}(\overline{\bar{Q}}}{\overline{\dot{Q}}}\right.$

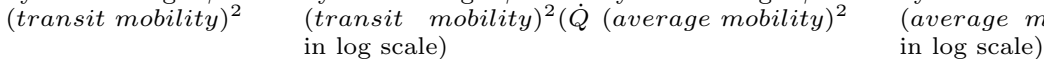
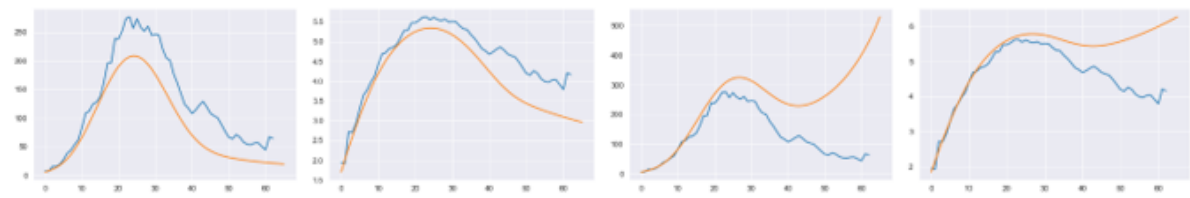

Figure 60: Fitting of Figure 61: Fitting of Figure 62: Fitting of Figure 63: Fitting of $\dot{Q}$ for Czech Repub- $\dot{Q}$ for Czech Repub- $\dot{Q}$ for Czech Repub- $\dot{Q}$ for Czech Repub-

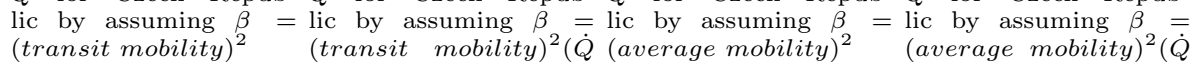
in $\log$ scale) in $\log$ scale)
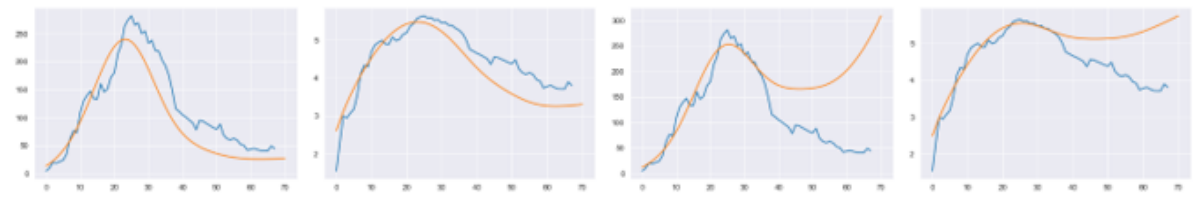

Figure 64: Fitting Figure 65: Fitting Figure 66: Fitting Figure 67: Fitting of $\dot{Q}$ for Norway of $\dot{Q}$ for Norway of $\dot{Q}$ for Norway of $\dot{Q}$ for Norway by assuming $\beta=$ by assuming $\beta=$ by assuming $\beta=$ by assuming $\beta=$

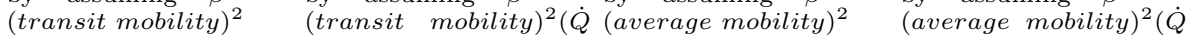
in $\log$ scale)

in $\log$ scale) 
medRxiv preprint doi: https://doi.org/10.1101/2021.01.11.20248882; this version posted January 15, 2021. The copyright holder for this preprint (which was not certified by peer review) is the author/funder, who has granted medRxiv a license to display the preprint in perpetuity.

\section{It is made available under a CC-BY 4.0 International license .}
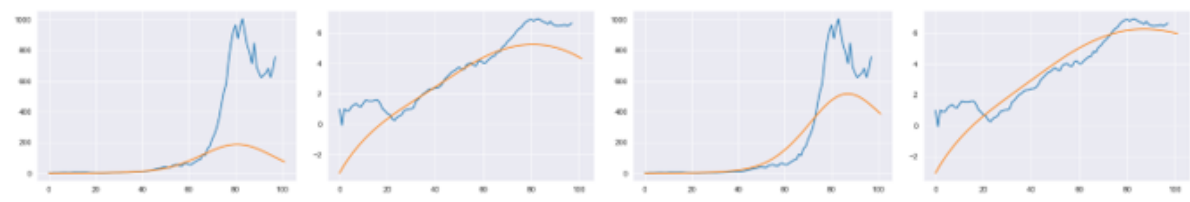

Figure 68: Fitting Figure 69: Fitting Figure 70: $\quad$ Fitting Figure 71: Fitting of $\dot{Q}$ for Singapore of $\dot{Q}$ for Singapore of $\dot{Q}$ for Singapore of $\dot{Q}$ for Singapore by assuming $\beta=$ by assuming $\beta=$ by assuming $\beta=$ by assuming $\beta=$

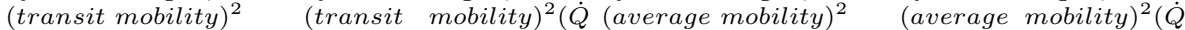
in $\log$ scale) in $\log$ scale)
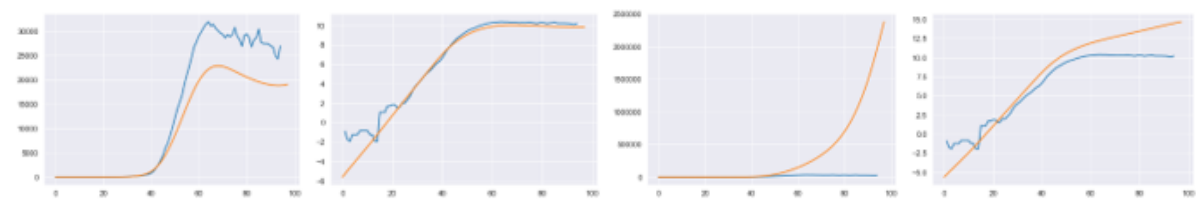

Figure 72: Fitting of Figure 73: Fitting of Figure 74: Fitting of Figure 75: Fitting of $\dot{Q}$ for United States $\dot{Q}$ for United States $\dot{Q}$ for United States $\dot{Q}$ for United States $\begin{aligned} & \text { by assuming } \beta=\text { by assuming } \beta=\text { by assuming } \beta= \\ & \text { (transit mobility })^{2}\end{aligned}=\underset{\text { by ansit mobility })^{2}(\dot{\dot{Q}} \text { (average mobility })^{2}}{\text { (average mobility })^{2}(\overline{\bar{Q}}}$ in $\log$ scale $) \quad$ in log scale)
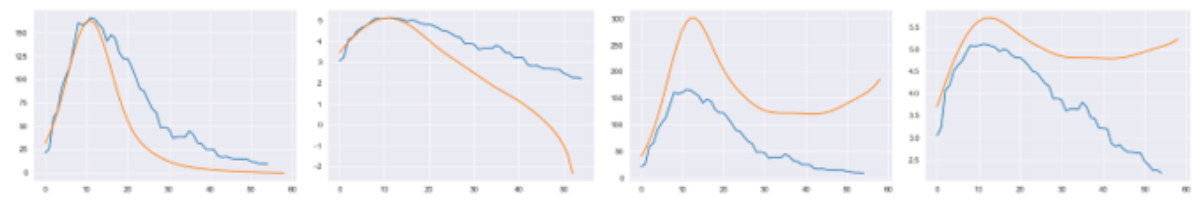

Figure 76: Fitting of Figure 77: Fitting of Figure 78: Fitting of Figure 79: Fitting of $\dot{Q}$ for Luxembourg $\dot{Q}$ for Luxembourg $\dot{Q}$ for Luxembourg $\dot{Q}$ for Luxembourg by assuming $\beta=$ by assuming $\beta=$ by assuming $\beta=$ by assuming $\beta=$

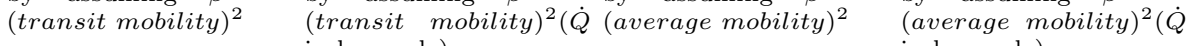
in $\log$ scale) in $\log$ scale) 


\begin{tabular}{|c||r|r|r|r|}
\hline country & $I_{0}$ & $\beta_{0}$ & $t_{0}$ & $\lambda_{2}$ \\
\hline \hline Australia & 0.008 & 0.7 & $2 / 2$ & 11 \\
Japan & 1.1 & 0.23 & $1 / 30$ & 13.3 \\
Czech & 80 & 0.4 & $3 / 6$ & 24 \\
Norway & 350 & 0.2 & $3 / 1$ & 20 \\
Switzerland & 100 & 0.6 & $2 / 29$ & 15 \\
Singapore & 4 & 0.35 & $1 / 30$ & 30 \\
United States & 0.04 & 0.4 & $2 / 3$ & 24 \\
Luxembourg & 60 & 1.3 & $3 / 13$ & 12 \\
\hline
\end{tabular}

Table 2: The parameters when we fit delayed SEIR model with assumption $\beta=$ (mobility of transit) ${ }^{2}$.

\section{Conclusion and Discussion}

In the above discussion, as a crude data analysis, we have seen that growth rate of confirmed cases of COVID-19 is roughly linear in activity index, with time delay around $10 \pm 5$ days. In a more refined data analysis, we have tried to fit to the data with SEIR model and similar model with time delay and seen that it is not likely SEIR fits the real data, while the model with time delay can reproduce the data. In that analysis, we saw that the the ratio of the value of contact coefficient $\beta$ before and after lockdown, $\beta_{1} / \beta_{0}$, is roughly equal to the ratio of square of mobility index before and after lockdown, so in the third analysis of this note, we have tried to fit the data with the assumption $\beta=(\text { mobility })^{2}$, using two different definition of mobility. We have confirmed that the data roughly fits this assumption, although the true value of contact coefficient should be in between the result expected by using average mobility, and the one by transit mobility. We need further investigation to know more rigorous relationship between the mobility and the contact coefficient for modelling the number of confirmed cases of COVID-19. In particular, if we wish to fit the parameters for the number of confirmed cases, we might not be able to fit well with the data for the second wave, or third wave of covid-19, so it seems we need other parameters than mobility to explain the dynamics of confirmed cases. In Japanese case, temperature could be a promising candidate for the explanatory parameter, because in the mid-late summer we saw the decrease in the number of confirmed cases.

\section{A Appendix}

In this appendix, we will see the data fitting for SEIR model without delay. Please compare this result with the delayed SEIR model we have seen above(Table 1 and Figure 12 Figure 51). The curve was fitted by hand and subject to some error. The parameters for the fitting are as in Table 3. We can see that in most of the countries delayed SEIR model fits better with the same number of parameters. 


\begin{tabular}{|c||r|r|r|r|r|r|r|r|r|r|}
\hline country & $\beta_{0}$ & $\beta_{1} / \beta_{0}$ & $\gamma$ & $\delta$ & $I_{0}$ & $t_{0}$ & $t_{1}$ & $t_{2}$ & $t_{2}-t_{1}$ & error \\
\hline \hline Australia & 0.8 & 0.01 & $1 / 4$ & $1 / 13$ & 120 & $3 / 1$ & $3 / 9$ & $3 / 20$ & 11 & 28.9 \\
Austria & 1 & 0.02 & $1 / 4$ & $1 / 5$ & 0.006 & $2 / 2$ & $3 / 12$ & $3 / 28$ & 16 & 1025.6 \\
Japan & 0.3 & 0.01 & $1 / 4$ & $1 / 17$ & 1.5 & $1 / 30$ & $3 / 24$ & $4 / 13$ & 20 & 415 \\
South Korea & 0.75 & 0.01 & $1 / 4$ & $1 / 11$ & 17 & $2 / 1$ & $2 / 19$ & $2 / 26$ & 7 & 78.3 \\
Czech Republic & 0.7 & 0.0 & $1 / 4$ & $1 / 27$ & 300 & $3 / 6$ & $3 / 8$ & $3 / 20$ & 12 & 33.7 \\
Norway & 0.7 & 0.01 & $1 / 4$ & $1 / 17$ & 600 & $3 / 1$ & $3 / 5$ & $3 / 12$ & 7 & 34.3 \\
Switzerland & 0.6 & 0.0 & $1 / 4$ & $1 / 17$ & 500 & $2 / 29$ & $3 / 8$ & $3 / 19$ & 11 & 24.4 \\
Singapore & 0.3 & 0.03 & $1 / 4$ & $1 / 20$ & 1.0 & $1 / 20$ & $3 / 19$ & $4 / 10$ & 22 & 527.6 \\
United States & 1.0 & 0.07 & $1 / 4$ & $1 / 15$ & 0.04 & $2 / 3$ & $3 / 8$ & $3 / 22$ & 14 & 725.1 \\
Luxembourg & 1.5 & 0.01 & $1 / 4$ & $1 / 12$ & 1000 & $3 / 13$ & $3 / 5$ & $3 / 20$ & 15 & 16.9 \\
Vietnam & 0.4 & 0.01 & $1 / 4$ & $1 / 5$ & 3 & $2 / 5$ & $3 / 3$ & $4 / 1$ & 29 & 99.2 \\
\hline
\end{tabular}

Table 3: The parameters when we fit SEIR model to the data.
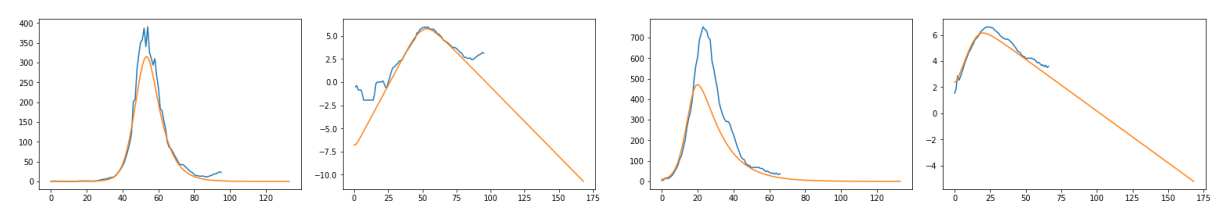

Figure 80: $\dot{Q}$ for actual Figure 81: $\dot{Q}$ for actual and SEIR model in Aus- and SEIR model in Australia tralia (in $\log$ scale)

Figure 82: $\dot{Q}$ for actual Figure 83: $\dot{Q}$ for actual and SEIR model in Aus- and SEIR model in Austria tria (in log scale)

The figures for the fitting are as in Figure $80 \sim$ Figure 101.

\section{References}

[1] Hethcote, Herbert W. "The mathematics of infectious diseases." SIAM review 42, no. 4 (2000): 599-653.

[2] COVID-19 情報共有 — COVID19-Information sharing, 都道府県ごとのシ ミュレーションによる検討, Sato, Aki-Hiro, et al. ”An epidemic simulation with a delayed stochastic SIR model based on international socioeconomic-
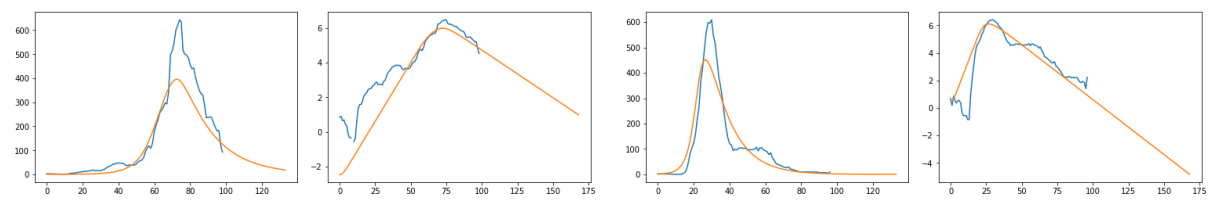

Figure 84: $\dot{Q}$ for ac- Figure 85: $\dot{Q}$ for actual and SEIR model in tual and SEIR model in Japan Japan (in log scale)

Figure 86: $\dot{Q}$ for actual Figure 87: $\dot{Q}$ for actual and SEIR model in Ko- and SEIR model in Ko-
rea 


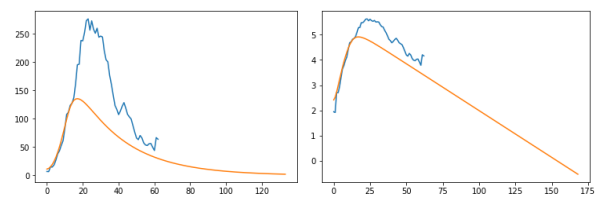

Figure 88: $\dot{Q}$ for ac- Figure 89: $\dot{Q}$ for actual and SEIR model in tual and SEIR model in Czech

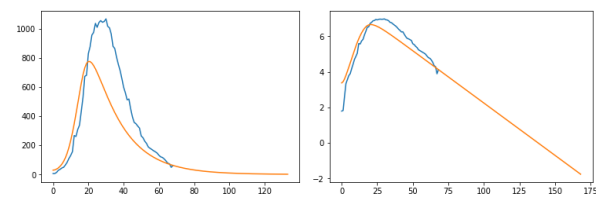

Figure 92: $\dot{Q}$ for ac- Figure 93: $\dot{Q}$ for actual and SEIR model in tual and SEIR model Switzerland in Switzerland (in log scale)
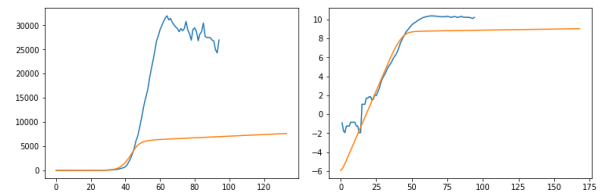

Figure 96: $\dot{Q}$ for ac- Figure 97: $\dot{Q}$ for actual and SEIR model in tual and SEIR model United States in United States (in log scale)
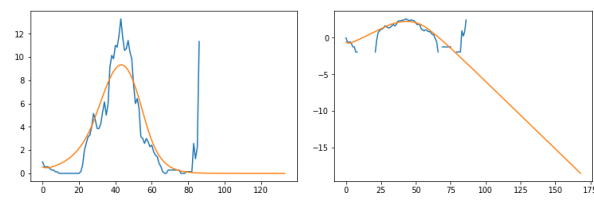

Figure 100: $\dot{Q}$ for ac- Figure 101: $\dot{Q}$ for actual and SEIR model in tual and SEIR model in Vietnam Vietnam (in log scale)

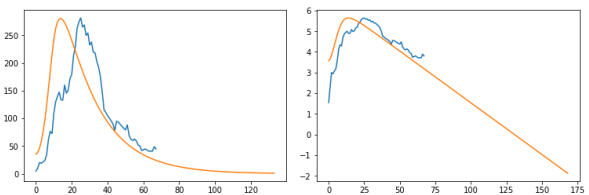

Figure 90: $\dot{Q}$ for actual Figure 91: $\dot{Q}$ for actual and SEIR model in Nor- and SEIR model in Norway way (in log scale)

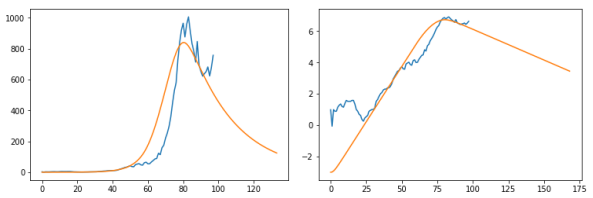

Figure 94: $\dot{Q}$ for actual Figure 95: $\dot{Q}$ for actual and SEIR model in Sin- and SEIR model in Singapore gapore (in log scale)

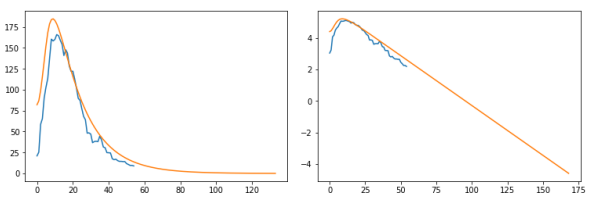

Figure 98: $\dot{Q}$ for actual Figure 99: $\dot{Q}$ for actual and SEIR model in Lux- and SEIR model in Luxembourg embourg (in log scale) 
technological databases." 2015 IEEE International Conference on Big Data (Big Data). IEEE, 2015.

[3] Mizumoto, Kenji, et al. "Estimating the asymptomatic proportion of coronavirus disease 2019 (COVID-19) cases on board the Diamond Princess cruise ship, Yokohama, Japan, 2020." Eurosurveillance 25.10 (2020).

[4] "Suspected patients wait up to a week to get tested ", https://www3.nhk.or.jp/nhkworld/en/news/20200417_33/

[5] https://ourworldindata.org/coronavirus

[6] https://www.apple.com/covid19/mobility

[7] https://twitter.com/shunyielong, https://twitter.com/ma_press, https://twitter.com/sken20k

[8] Wei, Wycliffe E. "Presymptomatic Transmission of SARS-CoV-2Singapore, January 23-March 16, 2020." MMWR. Morbidity and Mortality Weekly Report 69 (2020).

[9] https://datastudio.google.com/u/0/reporting/c4e0fe88-f72e-464e-a3b85e4e591c238d/page/ultJB?s=oA3tV-uQzaE 